\title{
Large Eddy Simulation of gravity currents with a high order DG method
}

\author{
Caterina Bassi $^{1 *}$, Antonella Abbà ${ }^{2}$, Luca Bonaventura ${ }^{1}$, \\ Lorenzo Valdettaro ${ }^{1}$ \\ ${ }^{1} \mathrm{MOX}$ - Modelling and Scientific Computing, Dipartimento di Matematica \\ Politecnico di Milano, Milan, Italy \\ ${ }^{2}$ Dipartimento di Scienze e Tecnologia Aerospaziali \\ Politecnico di Milano, Milan, Italy \\ *Email address for correspondence: caterina.bassi@polimi.it \\ Communicated by Enrico De Bernardis \\ Received on 12 20, 2016. Accepted on 05 29, 2017.
}

\begin{abstract}
This work deals with Direct Numerical Simulations (DNS) and Large Eddy Simulations (LES) of a turbulent gravity current in a gas, performed by means of a Discontinuous Galerkin (DG) Finite Elements method employing, in the LES case, LES-DG turbulence models previously introduced by the authors. Numerical simulations of non-Boussinesq lock-exchange benchmark problems show that, in the DNS case, the proposed method allows to correctly reproduce relevant features of variable density gas flows with gravity. Moreover, the LES results highlight, also in this context, the excessively high dissipation of the Smagorinsky model with respect to the Germano dynamic procedure.

Keywords: Large Eddy Simulation, dynamical models, density currents, low Mach number flows, Discontinuous Galerkin method.

AMS subject classification: 65M60,65Z05,76F25,76F50,76F65.
\end{abstract}

\section{Introduction}

Gravity currents are frequently encountered in geophysical flows, when a heavier fluid propagates into a lighter one in a predominantly horizontal direction because of the difference in hydrostatic pressure at the boundary between the two fluids. In atmospheric gravity currents, such as thunderstorm outflows, density difference is typically caused by the temperature difference between the cold front and the warmer surrounding air. In oceanic flows, density difference is caused by salinity and temperature gradients, while in pyroclastic flows the density difference is due to the presence of suspended particles in the flow. The evolution of gravity currents is also very important for some engineering applications, such as the accidental leakage of industrial gases, see e.g. [1].

In gravity currents, the density difference between the lighter and heavier fluid can range from very small to very large. In case of small density differences, density variations in the momentum equation can be neglected in the inertia term, but retained in the buoyancy term. This approximation is called Boussinesq approximation, see e.g. [2], and is sufficiently accurate for density differences up to a few percent. In most experimental 


\section{LES of gravity currents with high order DG method}

and computational studies of gravity currents reported in the literature, the Boussinesq approximation has been employed. However, in several of the above listed phenomena non-Boussinesq effects become important.

A wide range of interesting and important turbulent phenomena arise in gravity driven currents, such as breaking internal waves and Kelvin-Helmholtz instabilities. While simple algebraic closures have long been employed in many applications, see e.g. [3], a number of recent studies have been devoted to LES of Boussinesq gravity currents, see e.g. [4], [5], [6]. However, to the best of our knowledge, no published results are available in the non-Boussinesq case.

In the present work, we present a first validation of the Large Eddy Simulation model recently proposed in [7] applied to the study of turbulent gravity currents in a gas. This model is based on a high order, Discontinuous Galerkin finite element discretization. Such a numerical framework allows to generalize the concept of LES filter as a projection onto the polynomial space related to the discretization, thus making it possible to apply it to arbitrary unstructured meshes. This is conceptually close to what is done in Variational Multi Scale (VMS) models, see e.g. [8], [9]. The LES model employed in [7], however, was also the first to combine a VMS approach with more complex dynamical models for the subgrid stresses. Furthermore, we model gravity currents via the full Navier-Stokes equations for a compressible fluid, thus allowing for the simulation of both Boussinesq and non-Boussinesq gravity currents. In the limit of very low Mach number, this entails a loss of efficiency for the explicit time discretization presently employed in the [7] model. For this reason, a semi-implicit discretization is presently being developed. In this work, however, rather than on computational efficiency considerations we will focus on the performance of the turbulence model.

We provide a first assessment of the ability of our DG-LES model to reproduce the incompressible results obtained in [10]. The benchmark considered is the canonical lockexchange test case. It appears to be particularly interesting because it has been widely investigated both experimentally and numerically and, moreover, it provides a quite complex flow evolution (with the presence of shear driven mixing and internal waves), while being specified by simple and unambiguous forcing and initial/boundary conditions, see e.g. the discussion in [6]. We consider the lock-exchange benchmark in the non-Boussinesq regime, carrying out first simulations where all the turbulent scales of motion are correctly captured by the computational mesh (Direct Numerical Simulations). We then present results of simulations at higher Reynolds number on a mesh that is too coarse for complete resolution of all turbulent scales. Large Eddy Simulations have been carried out with the classic Smagorinsky model and with the Germano dynamic model. The numerical results reported in section 5 show that the method proposed in [7] is able to reproduce correctly all the relevant features of this important benchmark. The performance of the Smagorinsky model confirms the finding of [7] that also VMS approaches can benefit substantially from the use of more sophisticated subgrid stress models. On the other hand, the results also highlights the need for more detailed turbulence statistics than the bulk dissipation rate for a complete assessment of the LES models performance.

The paper is organized as follows. In section 2, the general model problem is introduced. Section 3 is devoted to the description of the turbulence models that have been applied. The space and time discretizations employed and their link with the proposed turbulence modeling approaches are presented in section 4 . The results of the numerical simulations are presented in section 5, while some conclusions and perspectives for future work are discussed in section 6 . 


\section{Bassi, Abbà, Bonaventura, Valdettaro}

\section{The model problem}

We consider the compressible Navier-Stokes equations, which can be written in dimensional form (denoted by the superscript "*"), employing the Einstein notation, as:

$$
\begin{aligned}
& \partial_{t}^{*} \rho^{*}+\partial_{x_{j}}^{*}\left(\rho^{*} u_{j}^{*}\right)=0 \text {, } \\
& \partial_{t}^{*}\left(\rho^{*} u_{i}^{*}\right)+\partial_{x_{j}}^{*}\left(\rho^{*} u_{i}^{*} u_{j}^{*}\right)+\partial_{x_{i}}^{*} p^{*}-\partial_{x_{j}}^{*} \sigma_{i j}^{*} \\
& =\rho^{*} f_{i}^{*} \text {, } \\
& \partial_{t}^{*}\left(\rho^{*} e^{*}\right)+\partial_{x_{j}^{*}}\left(\rho^{*} h^{*} u_{j}^{*}\right)-\partial_{x_{j}}^{*}\left(u_{i}^{*} \sigma_{i j}^{*}\right)+\partial_{x_{j}}^{*} q_{j}^{*} \\
& =\rho^{*} f_{j}^{*} u_{j}^{*},
\end{aligned}
$$

where $\rho^{*}, \mathbf{u}^{*}$ and $e^{*}$ denote density, velocity and specific total energy, respectively, $p^{*}$ is the pressure, $\mathbf{f}^{*}$ is a prescribed forcing (in the present investigation the gravity forcing is considered and we have $\left.\mathbf{f}^{*}=\left(0,0,-g^{*}\right)\right), h^{*}$ is the specific enthalpy, defined by $\rho^{*} h^{*}=$ $\rho^{*} e^{*}+p^{*}$, and $\sigma^{*}$ and $\mathbf{q}^{*}$ are the diffusive momentum and heat fluxes. Equation (1) must be complemented with the state equation

$$
p^{*}=\rho^{*} R^{*} T^{*},
$$

where $T^{*}$ is the temperature and $R^{*}$ is the ideal gas constant. The temperature can then be expressed in terms of the prognostic variables introducing the specific internal energy $e_{\mathrm{i}}^{*}$, so that

$$
e^{*}=e_{\mathrm{i}}^{*}+\frac{1}{2} u_{k}^{*} u_{k}^{*}, \quad T^{*}=\frac{e_{\mathrm{i}}^{*}}{c_{v}^{*}},
$$

where $c_{v}^{*}$ is the specific heat at constant volume. Finally, the model is closed with the constitutive equations for the diffusive fluxes:

$$
\sigma_{i j}^{*}=\mu^{*} \mathcal{S}_{i j}^{d, *}, \quad q_{i}^{*}=-\frac{\mu^{*} c_{p}^{*}}{\operatorname{Pr}} \partial_{x_{i}}^{*} T^{*},
$$

where $\mathcal{S}_{i j}^{*}=\partial_{x_{j}}^{*} u_{i}^{*}+\partial_{x_{i}}^{*} u_{j}^{*}$ and $\mathcal{S}_{i j}^{d, *}=\mathcal{S}_{i j}^{*}-\frac{1}{3} \mathcal{S}_{k k}^{*} \delta_{i j}, c_{p}^{*}=R^{*}+c_{v}^{*}$ is the specific heat at constant pressure, $\operatorname{Pr}$ denotes the Prandtl number, and the dynamic viscosity $\mu^{*}$ is assumed to depend only on temperature $T^{*}$ according to the power law

$$
\mu^{*}\left(T^{*}\right)=\mu_{0}^{*}\left(\frac{T^{*}}{T_{0}^{*}}\right)^{\alpha}
$$

following Sutherland's hypothesis (see e.g. [11]). The dimensionless form of the problem is obtained assuming four reference quantities: $\rho_{r}, L_{r}, V_{r}$ and $T_{r}$. All the other reference quantities are derived from these fundamental ones by means of dimensional considerations (see Table 1). As a result, the following non dimensional form will be used:

$$
\begin{aligned}
& \partial_{t} \rho+\partial_{j}\left(\rho u_{j}\right)=0, \\
& \partial_{t}\left(\rho u_{i}\right)+\partial_{j}\left(\rho u_{i} u_{j}\right)+\partial_{i} p-\partial_{j} \sigma_{i j}=\rho f_{i}, \\
& \partial_{t}(\rho e)+\partial_{j}\left(\rho h u_{j}\right)-\partial_{j}\left(u_{i} \sigma_{i j}\right) \\
& \quad+\partial_{j} q_{j}=\rho f_{j} u_{j},
\end{aligned}
$$

where $\mathbf{f}=\left(0,0,-1 / F r^{2}\right) . F r$ is the Froude number, which is related to the nondimensional gravity acceleration by the following equation:

$$
g=\frac{g^{*}}{g_{r}}=\frac{g^{*} L_{r}}{V_{r}^{2}}=\frac{1}{F r^{2}} .
$$




\section{LES of gravity currents with high order DG method}

Table 1. Reference physical quantitites

\begin{tabular}{ccc}
\hline Physical & $\begin{array}{c}\text { Reference } \\
\text { physical } \\
\text { quantities }\end{array}$ & $\begin{array}{c}\text { Fundamental } \\
\text { physical } \\
\text { quantities }\end{array}$ \\
\hline Length & $L_{r}$ & - \\
Density & $\rho_{r}$ & - \\
Velocity & $V_{r}$ & - \\
Temperature & $T_{r}$ & - \\
Pressure & $p_{r}$ & $\rho_{r} V_{r}^{2}$ \\
Gas constant & $R_{r}$ & $V_{r}^{2} / T_{r}$ \\
Time & $t_{r}$ & $L_{r} / V_{r}$ \\
Internal energy & $e_{r}$ & $V_{r}^{2}$ \\
Dynamic viscosity & $\mu_{r}$ & $\rho_{r} L_{r} V_{r}$ \\
Thermal conductivity & $\lambda_{r}$ & $\rho_{r} V_{r}^{3} L_{r} / T_{r}$ \\
Specific heat (const. vol.) & $c_{v, r}$ & $V_{r}^{2} / T_{r}$ \\
Specific heat (const. press.) & $c_{p, r}$ & $V_{r}^{2} / T_{r}$ \\
\hline
\end{tabular}

Some other important quantities and equations in non-dimensional form are derived in the following. The non-dimensional form of the gas constant is given by:

$$
R=\frac{R^{*}}{R_{r}}=\frac{R^{*} T_{r}}{V_{r}^{2}}=\frac{1}{\gamma M a^{2}}
$$

where $M a$ is the Mach number and $\gamma$ the ratio between specific heats. The nondimensional specific heat at constant volume is:

$$
c_{v}=\frac{c_{v}^{*}}{c_{v, r}}=\frac{c_{v}^{*} T_{r}}{V_{r}^{2}}=\frac{R^{*} T_{r}}{(\gamma-1) V_{r}^{2}}=\frac{1}{\gamma(\gamma-1) M a^{2}} .
$$

Using equations (8) and (9) we obtain for the specific heat at constant pressure: $c_{p}=$ $1 /(\gamma-1) M a^{2}$. The non-dimensional form of the state equation is obtained starting from (2). Expressing the dimensional quantities as a function of the non-dimensional and reference ones we have:

$$
\rho_{r} V_{r}^{2} p=\rho_{r} \rho \frac{V_{r}^{2}}{T_{r}} R T_{r} T
$$

dividing by $\rho_{r} V_{r}^{2}$ and using equation (8) we obtain:

$$
p=\rho R T=\frac{\rho T}{\gamma M a^{2}} .
$$

Starting from the dimensional equation (3), the non-dimensional internal energy is obtained as:

$$
e_{i}=\frac{T}{(\gamma-1) \gamma M a^{2}}
$$

while the total energy is:

$$
e=e_{\mathrm{i}}+\frac{1}{2} u_{k} u_{k} \text {. }
$$




\section{Bassi, Abbà, Bonaventura, Valdettaro}

The dynamic viscosity $\mu$ is given by:

$$
\mu=\frac{\mu^{*}}{\mu_{r}}=\frac{\mu_{0}^{*}}{\rho_{r} L_{r} V_{r}}\left(\frac{T_{r} T}{T_{0}^{*}}\right)^{\alpha}=\frac{1}{R e} T^{\alpha},
$$

where the last equality holds because we set $T_{0}^{\mathrm{d}}=T_{r}$. In [10] the non-dimensional dynamic viscosity is:

$$
\mu=\frac{\rho}{R e}
$$

Since in our simulations the pressure field is substantially constant during the computation, this dependency upon density can be reproduced by retaining the Sutherland law (14) and by setting the parameter $\alpha=-1$. Alternatively equation (15) can be directy employed. The thermal conductivity $\lambda$ is given by $\lambda=\mu c_{p} / \operatorname{Pr}$, while the nondimensional constitutive equations for the diffusive fluxes are $\sigma_{i j}=\mu \mathcal{S}_{i j}^{d}, q_{i}=-\lambda \partial_{i} T$, with $\mathcal{S}_{i j}=\partial_{j} u_{i}+\partial_{i} u_{j}$ and $\mathcal{S}_{i j}^{d}=\mathcal{S}_{i j}-\frac{1}{3} \mathcal{S}_{k k} \delta_{i j}$.

\section{The LES model}

We will present here a compact description of the LES models employed in this paper. We refer to [12] for a general introduction to LES modelling and to [7] for a more complete description of these and other LES models. As it is well known, the key ingredient of a LES model is the filtering operator. In the approach proposed in [7], the filter operator is embedded in the spatial DG discretization. The details of this realization of the filter operator will be given in section 4 . Here, we only anticipate that the filtering operator is denoted by ${ }^{-}$and that it is associated with the spatial scale $\Delta$. The spatial scale depends on the local element size and is as a consequence a piecewise constant function in space.

As customary in LES of compressible flows, see e.g. [13], we introduce also the Favre filtering operator $\widetilde{*}$ which is defined implicitly by the Favre decomposition. Given a generic function $f$, the Favre decomposition is defined as:

$$
\overline{\rho f}=\bar{\rho} \tilde{f}
$$

This decomposition is introduced for velocity, total energy, internal energy, enthalpy and temperature, yielding the equations

$$
\begin{aligned}
\overline{\rho u_{i}} & =\bar{\rho} \widetilde{u}_{i}, \\
\overline{\rho e} & =\bar{\rho} \widetilde{e}=\bar{\rho} \widetilde{e_{i}}+\frac{1}{2}\left(\bar{\rho} \widetilde{u}_{k} \widetilde{u}_{k}+\tau_{k k}\right), \\
\overline{\rho e_{i}} & =\bar{\rho} \widetilde{e_{i}}=\frac{1}{\gamma(\gamma-1) M a^{2}} \bar{\rho} \widetilde{T}, \\
\overline{\rho h} & =\bar{\rho} \widetilde{h}=\bar{\rho} \widetilde{e}+\bar{p}, \\
\overline{\rho T} & =\bar{\rho} \widetilde{T}=\gamma M a^{2} \bar{p},
\end{aligned}
$$

where the last equality in (17b) holds because of equation (13) while the last equalities in equations $(17 \mathrm{c})$ and $(17 \mathrm{e})$ follow from the state equation written in the usual form (eq. (11)) and in the internal energy form (eq. (12)) respectively. Notice that $\tau_{k k}$ in equation (17b) is the trace of the subgrid stress tensor which is defined as:

$$
\tau_{i j}=\overline{\rho u_{i} u_{j}}-\bar{\rho} \widetilde{u}_{i} \widetilde{u}_{j}
$$




\section{LES of gravity currents with high order DG method}

We introduce also the filtered counterpart of the diffusive fluxes:

$$
\widetilde{\sigma}_{i j}=\mu \widetilde{\mathcal{S}}_{i j}^{d}, \quad \widetilde{q}_{i}=-\lambda \partial_{i} \widetilde{T},
$$

with $\widetilde{\mathcal{S}}_{i j}=\partial_{j} \widetilde{u}_{i}+\partial_{i} \widetilde{u}_{j}$ and $\widetilde{\mathcal{S}}_{i j}^{d}=\widetilde{\mathcal{S}}_{i j}-\frac{1}{3} \widetilde{\mathcal{S}}_{k k} \delta_{i j}$. Given these definitions, the filter operator is applied to the non-dimensional form of the Navier-Stokes equations (6), thus obtaining:

$$
\begin{aligned}
& \partial_{t} \bar{\rho}+\partial_{j}\left(\bar{\rho} \widetilde{u}_{j}\right)=0 \\
& \partial_{t}\left(\bar{\rho} \widetilde{u}_{i}\right)+\partial_{j}\left(\bar{\rho} \widetilde{u}_{i} \widetilde{u}_{j}\right)+\partial_{i} \bar{p}-\partial_{j} \widetilde{\sigma}_{i j} \\
& =-\partial_{j} \tau_{i j}-\partial_{j} \epsilon_{i j}^{\mathrm{sgs}}+\bar{\rho} f_{i} \\
& \partial_{t}(\bar{\rho} \widetilde{e})+\partial_{j}\left(\bar{\rho} \widetilde{h} \widetilde{u}_{j}\right)-\partial_{j}\left(\widetilde{u}_{i} \widetilde{\sigma}_{i j}\right)+\partial_{j} \widetilde{q}_{j} \\
& =-\partial_{j}\left(\rho h u_{j}\right)^{\mathrm{sgs}}+\partial_{j} \phi_{j}^{\mathrm{sgs}} \\
& -\partial_{j} \theta_{j}^{\mathrm{sgs}}+\bar{\rho} f_{j} \widetilde{u}_{j} \text {, }
\end{aligned}
$$

where

$$
\begin{aligned}
& \epsilon_{i j}^{\mathrm{sgs}}=\bar{\sigma}_{i j}-\widetilde{\sigma}_{i j}, \quad\left(\rho h u_{i}\right)^{\mathrm{sgs}}=\overline{\rho h u_{i}}-\bar{\rho} \widetilde{h} \widetilde{u}_{i}, \\
& \phi_{j}^{\mathrm{sgs}}=\overline{u_{i} \sigma_{i j}}-\widetilde{u}_{i} \widetilde{\sigma}_{i j}, \theta_{i}^{\mathrm{sgs}}=\bar{q}_{i}-\widetilde{q}_{i} .
\end{aligned}
$$

In the subgrid terms, the same simplifications are performed as in [7], to which we refer the reader for a more detailed discussion. As a result the filtered equations (20) become:

$$
\begin{aligned}
& \partial_{t} \bar{\rho}+\partial_{j}\left(\bar{\rho} \widetilde{u}_{j}\right)=0 \\
& \begin{aligned}
\partial_{t}\left(\bar{\rho} \widetilde{u}_{i}\right)+ & \partial_{j}\left(\bar{\rho} \widetilde{u}_{i} \widetilde{u}_{j}\right)+\partial_{i} \bar{p}-\partial_{j} \widetilde{\sigma}_{i j} \\
& =-\partial_{j} \tau_{i j}+\bar{\rho} f_{i}
\end{aligned} \\
& \begin{aligned}
\partial_{t}(\bar{\rho} \widetilde{e})+ & \partial_{j}\left(\bar{\rho} \widetilde{h} \widetilde{u}_{j}\right)-\partial_{j}\left(\widetilde{u}_{i} \widetilde{\sigma}_{i j}\right)+\partial_{j} \widetilde{q}_{j} \\
& =-\frac{1}{(\gamma-1) M a^{2}} \partial_{j} Q_{j}^{\mathrm{sgs}}-\frac{1}{2} \partial_{j}\left(J_{j}^{\mathrm{sgs}}-\tau_{k k} \widetilde{u}_{j}\right)+\bar{\rho} f_{j} \widetilde{u}_{j} .
\end{aligned}
\end{aligned}
$$

The term $\tau_{i j}$ in the momentum equation $(22 \mathrm{~b})$ and $Q_{j}^{\text {sgs }}$ and $J_{j}^{\text {sgs }}$ in energy conservation equation (22c) are subgrid terms and need modeling. In the present work both the classic Smagorinsky model and the Germano dynamic procedure [14] have been employed.

In Smagorinsky-type subgrid models, the deviatoric part of the subgrid stress tensor $\tau_{i j}$ in (22) is modelled by a scalar turbulent viscosity $\nu^{\text {sgs }}$ :

$$
\begin{aligned}
& \tau_{i j}-\frac{1}{3} \tau_{k k} \delta_{i j}=-\bar{\rho} \nu^{\mathrm{sgs}} \widetilde{\mathcal{S}}_{i j}^{d}, \\
& \nu^{\text {sgs }}=C_{S}^{2} \Delta^{2}|\widetilde{\mathcal{S}}|
\end{aligned}
$$

where $C_{S}=0.1$ is the Smagorinsky constant, $|\widetilde{\mathcal{S}}|^{2}=\frac{1}{2} \widetilde{\mathcal{S}}_{i j} \widetilde{\mathcal{S}}_{i j}$ and $\Delta$ is the filter scale. The isotropic part of the subgrid stress tensor can be modelled as in [7]:

$$
\tau_{k k}=C_{I} \bar{\rho} \Delta^{2}|\widetilde{\mathcal{S}}|^{2} .
$$

The subgrid temperature flux is set proportional to the resolved temperature gradient:

$$
Q_{i}^{\mathrm{sgs}}=-\frac{1}{\operatorname{Pr} \mathrm{sgs}} \bar{\rho} \nu^{\mathrm{sgs}} \partial_{i} \widetilde{T}
$$




\section{Bassi, Abbà, Bonaventura, Valdettaro}

where $P r^{\text {sgs }}$ is a subgrid Prandtl number. Finally the term $J_{i}^{\text {sgs }}$ is treated as in [7]:

$$
J_{i}^{\mathrm{sgs}} \approx 2 \widetilde{u}_{k} \tau_{i k}+\widetilde{u}_{i} \tau_{k k} .
$$

In the Germano dynamic model [14], the terms $C_{S}$ and $C_{I}$ in the Smagorinsky model are no more chosen a priori for the whole domain, but are computed dynamically as functions of the resolved field. The deviatoric part of the stress tensor is the same as in the Smagorinsky model:

$$
\tau_{i j}-\frac{1}{3} \tau_{k k} \delta_{i j}=-\bar{\rho} C_{S} \Delta^{2}|\widetilde{\mathcal{S}}| \widetilde{\mathcal{S}}_{i j}^{d} .
$$

The coefficient $C_{S}$ is dynamically computed by introducing a test filter operator $\hat{\imath}$. This operator is linked to the numerical discretization and will be precisely defined in the next section; here it will suffice to point out that, as the filter operator ', the test filter is associated to a spatial scale $\widehat{\Delta}$ (larger than the spatial scale $\Delta$ associated to ${ }^{-}$). A Favre filter denoted with $\breve{r}$ is associated to the test filter through the following Favre decomposition:

$$
\widehat{\rho f}=\widehat{\rho} \breve{f} .
$$

If the test filter $\hat{\imath}$ is applied to the momentum equation $(22 \mathrm{~b})$ we obtain:

$$
\begin{array}{r}
\partial_{t}\left(\widehat{\rho} \breve{u}_{i}\right)+\partial_{j}\left(\widehat{\rho} \breve{u}_{i} \breve{u}_{j}\right)+\partial_{i} \widehat{p}-\partial_{j} \widehat{\sigma}_{i j} \\
=-\partial_{j}\left(\widehat{\tau}_{i j}+\mathcal{L}_{i j}\right),
\end{array}
$$

where

$$
\mathcal{L}_{i j}=\widehat{\bar{\rho} \widetilde{u}_{i} \widetilde{u}_{j}}-\widehat{\bar{\rho}} \breve{u}_{i} \breve{\widetilde{u}}_{j}
$$

is the Leonard stress tensor. We now assume that the deviatoric part of the Leonard stress tensor can be modelled using an eddy viscosity model:

$$
\widehat{\tau}_{i j}^{d}+\mathcal{L}_{i j}^{d}=-\widehat{\bar{\rho}} \widehat{\Delta}^{2}|\breve{\widetilde{\mathcal{S}}}| C_{S} \breve{\widetilde{\mathcal{S}}}_{r s} .
$$

Substituting (27) for $\tau_{i j}^{d}$ and using a least square approach we obtain for the Smagorinsky constant $C_{S}$ the following expression:

$$
C_{S}=\frac{\mathcal{L}_{i j}^{d} \mathcal{R}_{i j}}{\mathcal{R}_{k l} \mathcal{R}_{k l}}
$$

where $\mathcal{R}_{k l}=\bar{\rho} \Delta^{2|\widetilde{\mathcal{S}}|} \widetilde{\mathcal{S}}_{k l}^{d}-\widehat{\bar{\rho}} \widehat{\Delta}^{2}|\breve{\widetilde{\mathcal{S}}}| \breve{\mathcal{S}}_{k l}$. The same dynamic procedure is applied also to the isotropic component of the subgrid stress tensor as in [7]. A similar approach is proposed also for the subgrid terms in the energy equation. For the subgrid heat flux we obtain:

$$
Q_{i}^{\mathrm{sgs}}=-\bar{\rho} \Delta^{2}|\widetilde{\mathcal{S}}| C_{Q} \partial_{i} \widetilde{T} .
$$

After having applied a dynamic procedure we have:

$$
C_{Q}=\frac{\mathcal{L}_{i}^{Q} \mathcal{R}_{i}^{Q}}{\mathcal{R}_{k}^{Q} \mathcal{R}_{k}^{Q}},
$$

with $\mathcal{R}_{i}^{Q}=\bar{\rho} \Delta^{2 \mid \widetilde{\mathcal{S}}} \partial_{i} \widetilde{T}-\widehat{\bar{\rho}} \widehat{\Delta}^{2}|\breve{\widetilde{\mathcal{S}}}| \partial_{i} \breve{\widetilde{T}}$ and $\mathcal{L}_{i}^{Q}=\widehat{\bar{\rho} \widetilde{u}_{i} \widetilde{T}}-\widehat{\bar{\rho}} \breve{\breve{u}}_{i} \breve{\widetilde{T}}$ temperature Leonard flux. The subgrid turbulent diffusion flux is defined as in [7]:

$$
J_{i}^{\mathrm{sgs}}=-\bar{\rho} \Delta^{2}|\widetilde{\mathcal{S}}| C_{J} \partial_{i}\left(\frac{1}{2} \widetilde{u}_{k} \widetilde{u}_{k}\right)+2 \widetilde{u}_{k} \tau_{i k}+\widetilde{u}_{i} \tau_{k k} .
$$




\section{LES of gravity currents with high order DG method}

where $C_{J}$ is dinamically computed. It is important to point out that all the dynamic coefficients are averaged over each element in order to avoid numerical instabilities; moreover, since the dynamic model allows backscattering, a clipping procedure analogous to the one introduced in [7] is applied to ensure that the total dissipation, resulting from both the viscous and the subgrid stresses, is positive.

\section{Numerical method}

The equations introduced in section 2 , together with the subgrid scale models of section 3, are spatially discretized by the Discontinuous Galerkin finite elements method. The DG approach is analogous to that described in [15]. In particular the Local Discontinuous Galerkin (LDG) method is chosen for the approximation of the second order viscous terms (see [16], [17], [18], [19]). In the LDG method, the non-dimensional system of Navier-Stokes equations (22) is rewritten introducing an auxiliary variable $\mathcal{G}$, so that

$$
\begin{aligned}
\partial_{t} \mathbf{U}+\nabla \cdot \mathbf{F}^{\mathrm{c}}(\mathbf{U}) & =\nabla \cdot \mathbf{F}^{\mathrm{v}}(\mathbf{U}, \mathcal{G}) \\
& -\nabla \cdot \mathbf{F}^{\mathrm{sgs}}(\mathbf{U}, \mathcal{G})+\mathbf{S} \\
\mathcal{G} & -\nabla \boldsymbol{\varphi}=0,
\end{aligned}
$$

where $\mathbf{U}=\left[\bar{\rho}, \bar{\rho} \widetilde{\mathbf{u}}^{T}, \bar{\rho} \widetilde{e}\right]^{T}$ are the prognostic variables, $\varphi=\left[\widetilde{\mathbf{u}}^{T}, \widetilde{T}\right]^{T}$ are the variables whose gradients enter the viscous fluxes (19), as well as the turbulent ones and $\mathbf{S}$ represents the source terms. The fluxes in (36) are written in the following compact form:

$$
\begin{gathered}
\mathbf{F}^{\mathrm{c}}=[\bar{\rho} \widetilde{\mathbf{u}}, \bar{\rho} \widetilde{\mathbf{u}} \otimes \widetilde{\mathbf{u}}+\bar{p} \mathcal{I}, \bar{\rho} \widetilde{h} \widetilde{\mathbf{u}}]^{T}, \\
\mathbf{F}^{\mathrm{v}}=\left[0, \widetilde{\sigma}, \widetilde{\mathbf{u}}^{T} \widetilde{\sigma}-\widetilde{\mathbf{q}}\right]^{T},
\end{gathered}
$$

and

$$
\begin{gathered}
\mathbf{F}^{\mathrm{sgs}}=\left[0, \tau, \frac{1}{(\gamma-1) M a^{2}} \mathbf{Q}^{\mathrm{sgs}}+\frac{1}{2}\left(\mathbf{J}^{\mathrm{sgs}}-\tau_{k k} \widetilde{\mathbf{u}}\right)\right], \\
\mathbf{S}=[0, \bar{\rho} \mathbf{f}, \bar{\rho} \mathbf{f} \cdot \widetilde{\mathbf{u}}] .
\end{gathered}
$$

Here, $\tau, \mathbf{Q}^{\text {sgs }}$ and $\mathbf{J}^{\text {sgs }}$ are given by (23), (25) and (26), respectively, for the Smagorinsky model while they are given by (27), (33) and (35) for the dynamic model.

To define the space discretization, a tessellation $\mathcal{T}_{h}$ of $\Omega$ into tetrahedral elements $K$ such that $\Omega=\bigcup_{K \in \mathcal{T}_{h}} K$ and $K \cap K^{\prime}=\emptyset$ is introduced and the finite element space is defined as:

$$
\mathcal{V}_{h}=\left\{v_{h} \in L^{2}(\Omega):\left.v_{h}\right|_{K} \in \mathbb{P}^{q}(K), \forall K \in \mathcal{T}_{h}\right\}
$$

where $q$ is a nonnegative integer and $\mathbb{P}^{q}(K)$ denotes the space of polynomial functions of total degree at most $q$ on $K$. For each element, the outward unit normal on $\partial K$ will be denoted by $\mathbf{n}_{\partial K}$. Given $d$ the dimension of the problem the numerical solution is now defined as $\left(\mathbf{U}_{h}, \mathcal{G}_{h}\right) \in\left(\left(\mathcal{V}_{h}\right)^{(2+d)},\left(\mathcal{V}_{h}\right)^{4 \times d}\right)$ such that, $\forall K \in \mathcal{T}_{h}, \forall v_{h} \in \mathcal{V}_{h}, \forall \mathbf{r}_{h} \in\left(\mathcal{V}_{h}\right)^{d}$, 


\section{Bassi, Abbà, Bonaventura, Valdettaro}

$$
\begin{aligned}
\frac{d}{d t} \int_{K} \mathbf{U}_{h} v_{h} d \mathbf{x} & -\int_{K} \mathbf{F}\left(\mathbf{U}_{h}, \mathcal{G}_{h}\right) \cdot \nabla v_{h} d \mathbf{x} \\
& +\int_{\partial K} \mathbf{F}^{*}\left(\mathbf{U}_{h}, \mathcal{G}_{h}\right) \cdot \mathbf{n}_{\partial K} v_{h} d \sigma=\int_{K} \mathbf{S} v_{h} d \mathbf{x} \\
\int_{K} \mathcal{G}_{h} \cdot \mathbf{r}_{h} d \mathbf{x} & +\int_{K} \boldsymbol{\varphi}_{h} \nabla \cdot \mathbf{r}_{h} d \mathbf{x} \\
& -\int_{\partial K} \varphi^{*} \mathbf{n}_{\partial K} \cdot \mathbf{r}_{h} d \sigma=0
\end{aligned}
$$

where $\mathbf{U}_{h}=\left[\rho_{h}, \rho_{h} \mathbf{u}_{h}, \rho_{h} e_{h}\right]^{T}, \varphi_{h}=\left[\mathbf{u}_{h}, T_{h}\right]^{T}, \mathbf{F}=\mathbf{F}^{\mathrm{c}}-\mathbf{F}^{\mathrm{v}}+\mathbf{F}^{\mathrm{sgs}}$, and $\mathbf{F}^{*}, \varphi^{*}$ denote the so-called numerical fluxes. The numerical fluxes are responsible for the coupling among different elements. In this work we have tested a) the Rusanov flux, b) a modified version of the Rusanov flux appropriate for low Mach number flows, employing the velocity of the fluid as upwinding velocity c) the exact Godunov Riemann solver (implemented as in [20]). In order to avoid some numerical difficulties in simulations with small density differences (see the discussion in section 5.3), the last one has been extensively employed in the simulations for $\mathbf{F}^{*}$. The centered flux is employed for $\varphi^{*}$. On each element, the unknowns are expressed in terms of an orthogonal polynomial basis, yielding what is commonly called a modal DG formulation. All the integrals are evaluated using quadrature formulae from [21], which are exact for polynomial orders up to $2 q$. This results in a diagonal mass matrix in the time derivative term of (38) and simplifies the computation of $L^{2}$ projections to be introduced shortly in connection with the LES filters.

In the following the filter operators ${ }^{-}$and $\widehat{\cdot}$, introduced in section 3 , will be explicitly defined in the context of the DG finite elements method. In particular the filters operators are defined in terms of an $L^{2}$ projection, as suggested e.g. in [22], [23], [24]. Given a subspace $\mathcal{V} \subset L^{2}(\Omega)$, let $\Pi_{\mathcal{V}}: L^{2}(\Omega) \rightarrow \mathcal{V}$ be the associated projector defined by

$$
\int_{\Omega} \Pi_{\mathcal{V}} u v d \mathbf{x}=\int_{\Omega} u v d \mathbf{x}, \quad \forall u, v \in \mathcal{V}
$$

where the integrals are evaluated with the same quadrature rule used in (38). For $v \in$ $L^{2}(\Omega)$, the filter ${ }^{-}$is now defined by

$$
\bar{v}=\Pi_{\mathcal{V}_{h}} v
$$

Notice that the application of this filter is built in the discretization process and equivalent to it. Therefore, once the discretization of equations (36) has been performed, only : filtered quantities are computed by the model. To define the test filter, we then introduce

$$
\widehat{\mathcal{V}}_{h}=\left\{v_{h} \in L^{2}(\Omega):\left.v_{h}\right|_{K} \in \mathbb{P}^{\widehat{q}}(K), \forall K \in \mathcal{T}_{h}\right\}
$$

where $0 \leq \widehat{q}<q$, and we let, for $v \in L^{2}(\Omega)$,

$$
\widehat{v}=\Pi_{\widehat{\mathcal{V}}_{h}} v .
$$

By our previous identification of the ${ }^{-}$filter and the discretization, the quantities $\bar{\rho}, \bar{\rho} \widetilde{\mathbf{u}}$ and $\bar{\rho} \widetilde{e}$ can be identified with $\rho_{h}, \rho_{h} \mathbf{u}_{h}$ and $\rho_{h} e_{h}$, respectively. Therefore, they belong 


\section{LES of gravity currents with high order DG method}

to $\mathcal{V}_{h}$, for which an orthogonal basis is employed by the numerical method. As a result, the computation of $\widehat{\rho_{h}}, \widehat{\rho_{h} \mathbf{u}_{h}}$ and $\widehat{\rho_{h} e_{h}}$ is straightforward and reduces to zeroing the last coefficients in the local expansion. Assuming that the analytic solution is defined in some infinite dimensional subspace of $L^{2}$, heuristically, $\mathcal{V}_{h} \subset L^{2}$ is associated to the scales which are represented by the model, while $\widehat{\mathcal{V}}_{h} \subset \mathcal{V}_{h} \subset L^{2}$ is associated to the spatial scales well resolved by the numerical approximation.

The filtering operations (39) and (41) are realized by imposing pointwise the conditions (17), (18) and (19). The Leonard stress tensors are computed using (41) with the quadrature rules given in [21]. Notice that the filters defined by $L^{2}$ projections do not commute with differential operators. However, in this work we neglect the commutation errors.

The spatial scales $\Delta$ and $\widehat{\Delta}$ associated with the two filters (39) and (41) can be computed by dividing the element diameter by the cubic root (or the square root in two dimensions) of the number of degrees of freedom of $\mathbb{P}^{q}(K)$, for $\Delta$, and $\mathbb{P}^{\widehat{q}}(K)$, for $\widehat{\Delta}$; the filter scales are, as a consequence, piecewise constant functions in space. Finally, time integration was performed by a 4 stage explicit Runge-Kutta method.

All the computations were performed using the implementation of the above described method provided in the finite element library FEMilaro [25]. This tool exploits modern FORTRAN/MPI features, aims at providing a flexible environment for the development and testing of new finite element formulations and is publicly available under GPL license.

\section{The lock exchange benchmark}

The lock-exchange configuration used to assess the capability of the DG-LES model is represented in Figure 1. The domain length is $L=32$ and its height is $H=1$. A membrane at $x_{0}=14$ initially divides the rectangular container into two compartments. In our case, the two chambers are filled with the same fluid at different densities on the two sides of the membrane (higher density on the left and lower density on the right). Upon the removal of the membrane, the dense front moves rightward along the lower boundary, while the light front propagates leftward along the upper boundary.

The quantities chosen as reference physical quantities for nondimensionalization of the compressible NS equations are the height of the channel $H^{*}$, the larger of the two fluid densities $\rho_{1}^{*}$ and the buoyancy velocity $u_{b}^{*}=\sqrt{g^{\prime *} H^{*}}$. Notice that $g^{\prime *}$ is the reduced gravity, which is computed as:

$$
g^{\prime *}=g^{*} \frac{\rho_{1}^{*}-\rho_{2}^{*}}{\rho_{1}^{*}}
$$

where $g^{*}$ denotes the gravity acceleration and $\rho_{2}^{*}$ the lower density. Some relationships exist between the nondimensional numbers $F r$ and $M a$ and the characteristic quantities of the test case. For the Froude number Fr, the following equation is valid:

$$
\frac{1}{F r^{2}}=\frac{g^{*} H^{*}}{u_{b}^{* 2}}=\frac{g^{*}}{g^{*} \frac{\rho_{1}^{*}-\rho_{2}^{*}}{\rho_{1}^{*}}}=\frac{1}{1-\gamma_{r}},
$$

where $\gamma_{r}=\rho_{2} / \rho_{1}$ is the ratio between densities. If we write the definition of the Mach number as the ratio between the characteristic velocity (i.e., the buoyancy velocity) and the sound speed we obtain:

$$
M a^{2}=\frac{u_{b}^{* 2}}{\frac{\gamma p^{*}}{\rho_{1}^{*}}}=\frac{g^{*} H^{*}\left(\rho_{1}^{*}-\rho_{2}^{*}\right)}{\gamma p^{*}} .
$$




\section{Bassi, Abbà, Bonaventura, Valdettaro}

If we write the dimensional pressure $p^{*}=p p_{r}=p \rho_{1}^{*} u_{b}^{* 2}$ as the product of the nondimensional pressure multiplied by the reference pressure and we simplify, we obtain for the Mach number the following relationship:

$$
M a=\frac{1}{\sqrt{\gamma p}},
$$

where $\gamma$ is the ratio between the specific heats. Concerning the initial conditions, even though the DG finite element method would be able to manage a discontinuous initial datum, in order to better reproduce the results obtained in literature, the initial density profile is smoothed out as in [10]:

$$
\rho_{0}(x)=\frac{\gamma_{r}+1}{2}-\frac{1-\gamma_{r}}{2} \operatorname{erf}\left(\frac{x-x_{0}}{\sqrt{R e}}\right),
$$

where $x$ denotes the horizontal coordinate. Since we are considering the compressible Navier-Stokes equations, it is necessary to specify the initial conditions also for pressure and temperature. The initial pressure value at the top of the domain is computed by using equation (45) as $p_{i n}^{t o p}=1 /\left(\gamma M a^{2}\right)$. The initial pressure value in the whole domain is then computed assuming an hydrostatic pressure profile $p_{i n}=p_{i n}^{t o p}+\rho(x)(1-z) / F r^{2}$, where $z$ denotes the vertical coordinate. The initial datum for temperature is derived starting from density and pressure and using the equation of state. Since our aim is to reproduce incompressible results, a Mach number of 0.008 has been chosen. In order to

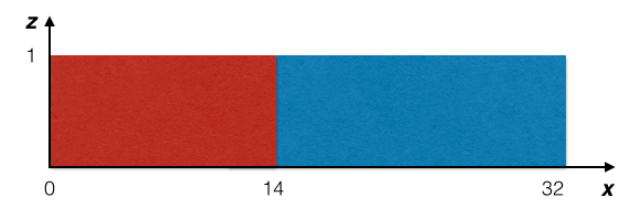

Figure 1. Initial datum for the lock-exchange configuration.

achieve effective comparisons with [10], two-dimensional simulations with slip boundary conditions have been performed. The performance of our model has been evaluated by comparing density contours and, more quantitatively, different kinds of energy budgets. In particular, we have computed the time evolution of the normalized potential, kinetic and dissipated energies integrated over the whole domain. The potential energy is:

$$
E_{p}(t)=\int_{\Omega} \frac{1}{F r^{2}} \rho z d V
$$

The kinetic energy is:

$$
E_{k}(t)=\int_{\Omega} \frac{1}{2} \rho u_{i} u_{i} d V
$$

while the time evolution of the dissipated energy is computed by solving the following equation:

$$
\frac{d E_{d}}{d t}=\int_{\Omega}\left\{\mu\left[\frac{1}{2}\left(\partial_{j} u_{i}+\partial_{i} u_{j}\right)^{2}-\frac{2}{3}(\nabla \cdot \mathbf{u})^{2}\right]\right\} d V .
$$

As proposed in [10] the potential energy of the light fluid $E_{a m b}$ is computed as:

$$
E_{a m b}=\frac{1}{1-\gamma_{r}} \int_{\Omega} \gamma_{r} z d V
$$




\section{LES of gravity currents with high order DG method}

From the initial potential energy, by subtracting $E_{a m b}$, we obtain $E_{p 0}^{a}=E_{p 0}-E_{a m b}$ (defined in [10] as initial available potential energy) and the available potential energy at a generic time $t E_{p}^{a}(t)=E_{p}(t)-E_{a m b}$. We then normalize each contribution to the overall energy budget with $E_{p 0}^{a}$ and we obtain the normalized potential energy $E_{p}^{n}(t)=E_{p}^{a}(t) / E_{p 0}^{a}$, the normalized kinetic energy $E_{k}^{n}(t)=E_{k}(t) / E_{p 0}^{a}$ and the normalized dissipated energy $E_{d}^{n}(t)=E_{d}(t) / E_{p 0}^{a}$. The second energy budget taken into consideration provides the evaluation of the energies dissipated by the dense and the light front separately, as suggested in [10]. The energies dissipated by the light and dense fronts are computed respectively integrating in time the following two equations:

$$
\begin{aligned}
& \left.\frac{d E_{d}^{\text {light }}(t)}{d t}=\int_{0}^{x_{0}} \int_{0}^{1} \mu\left[\frac{1}{2}\left(\partial_{j} u_{i}+\partial_{i} u_{j}\right)^{2}-\frac{2}{3}(\nabla \cdot \mathbf{u})^{2}\right]\right\} d z d x, \\
& \left.\frac{d E_{d}^{\text {dense }}(t)}{d t}=\int_{x_{0}}^{L} \int_{0}^{1} \mu\left[\frac{1}{2}\left(\partial_{j} u_{i}+\partial_{i} u_{j}\right)^{2}-\frac{2}{3}(\nabla \cdot \mathbf{u})^{2}\right]\right\} d z d x .
\end{aligned}
$$

where $x_{0}$ is the abscissa of the initial discontinuity.

\subsection{Direct Numerical Simulations}

For the fully resolved simulations, a grid composed by approximately 37000 elements has been employed. The polynomial degree has been set equal to 4 . Notice that, with these choices, the total number of degrees of freedom is of the same order of magnitude as that in [10].

In Figure 2 we compare the density contours obtained from our compressible NS simulations with the corresponding ones in [10], as computed at $t=10$ for density ratios $\gamma_{r}=0.2,0.7$ and for $R e=4000$. For both density ratios the results are quite similar to the reference solution, both in terms of the position of the front and of the number and appearance of the Kelvin-Helmoltz billows. In particular, considering the lower density ratio $\gamma_{r}=0.2$ (Figures 2(c)-2(d)) we notice that, as in [10], the dense front presents a considerably lower height and that, by $t=10$, it has propagated further with respect to the light front. Moreover, by decreasing the density ratio, we observe that the vortical structures appear to be confined to the region near the dense front. According to the explanation in [10], this is because across the dense current the velocity difference and the shear are larger, which has a destabilizing effect on the front. In the case of $\gamma_{r}=0.2$ we notice however some discrepancies between our results and those of [10]. The length of the area of the density current interested by the presence of Kelvin-Helmholtz billows is more extended in our case: the first vortex is located approximately at $x=13$ in our simulation (see Figure 2(c)) while its position is $x=15$ in [10] (Figure 2(d)).

Another important feature which is correctly reproduced by the DG simulations is the behaviour of the density current in the presence of constant dynamic viscosity (see Figure 3). In this situation the formation of vortices is limited to a smaller region close to the dense front (as highlighted in [10]). Considering energy budgets, in Figure 4(a) we present the time evolution of the normalized potential energy $E_{p}^{n}(t)$, the normalized kinetic energy $E_{k}^{n}(t)$ and the normalized dissipated energy $E_{d}^{n}(t)$ up to $t=10$, for $R e=4000$ and density ratio $\gamma_{r}=0.4$. We notice that the time evolution of the different energy budgets reported in [10] (dashed lines) is very well captured by the DG simulation (continuous lines). In Figure 4(b) we show the time evolution of the energies dissipated by the light and the dense front, respectively. Both in [10] (dashed lines) and in our DG simulation (continuous lines), the dense front (red lines) is more dissipative with respect to the light front (blue lines). However, a slight underestimation of the energy dissipated by the dense front is present in the DG simulation. 
Bassi, Abbà, Bonaventura, Valdettaro

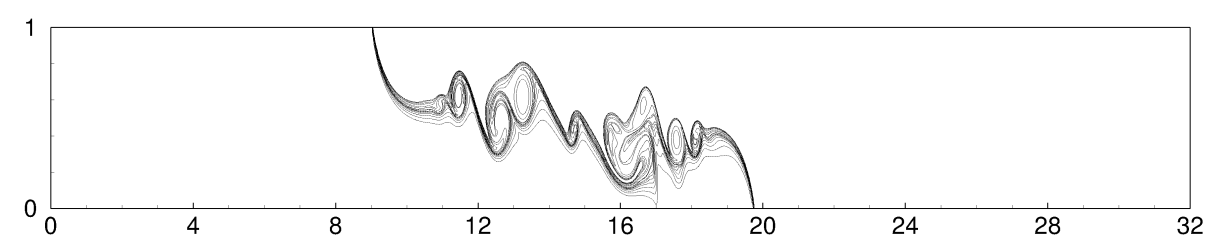

(a)

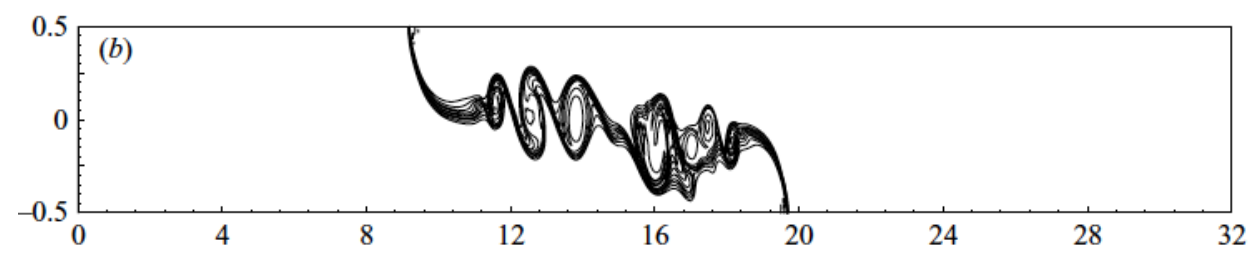

(b)

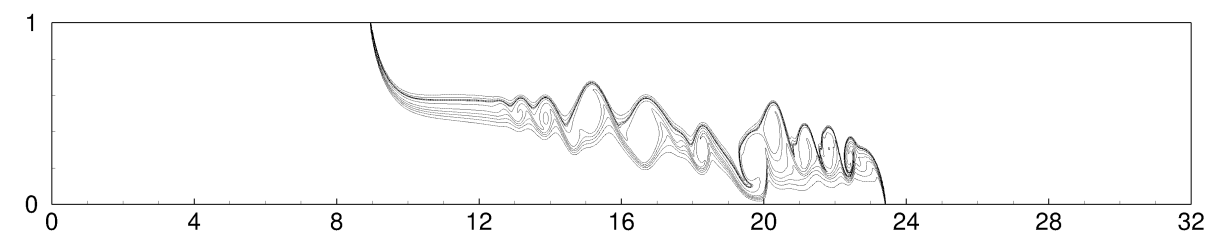

(c)

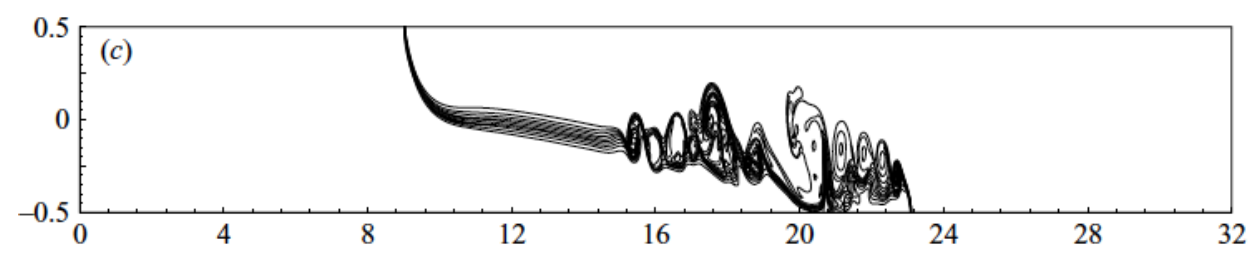

(d)

Figure 2. Density contours at $t=10$ for $R e=4000$ (DNS) (a) DG simulation, $\gamma_{r}=0.7$ (b) [10], $\gamma_{r}=0.7$ (c) DG simulation, $\gamma_{r}=0.2$ (d) [10], $\gamma_{r}=0.2$

\subsection{Large Eddy Simulations}

Besides simulations at DNS resolution, also some Large Eddy Simulation experiments have been carried out, using a density ratio $\gamma_{r}=0.7$ and Reynolds number $R e=40000$. Notice that, in these cases, no reference DNS results are available and no comparison with the results of [10] is attempted. These experiments have been performed employing the same computational grid as in the DNS at $R e=4000$ and polynomial degrees $p=2,3,4$. The polynomial degree associated to the test filter operation was taken to be 1 for $p=2$ 


\section{LES of gravity currents with high order DG method}

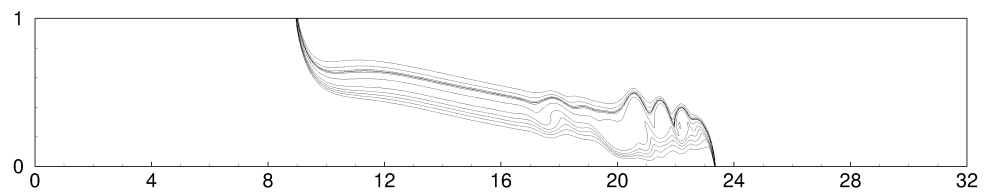

Figure 3. Density contours at $t=10$ for $\gamma_{r}=0.2$ and $R e=4000$ (DNS) with constant dynamic viscosity $\mu=1 / R e$.

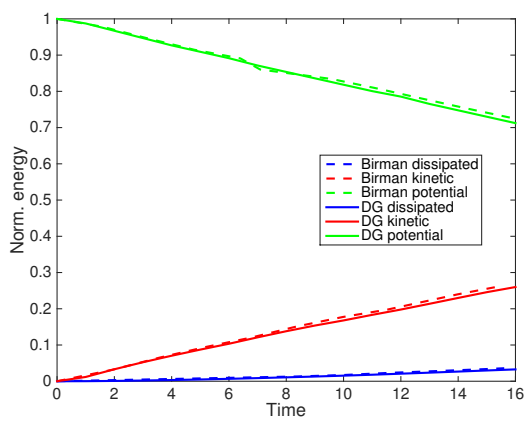

(a)

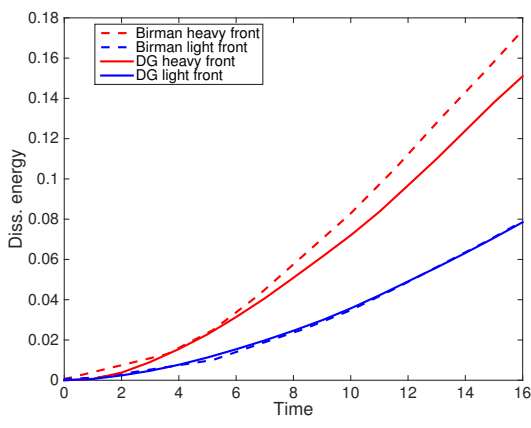

(b)

Figure 4. (a) Time evolution of the normalized potential energy $E_{p}^{n}$ (green), normalized kinetic energy $E_{k}^{n}$ (red) and normalized dissipated energy $E_{d}^{n}$ (blue) for $\gamma_{r}=0.4$ and $R e=4000$ (DNS). Continuous lines: DG simulation. Dashed lines: [10] simulation. (b) Time evolution of the energies dissipated by the dense (red) and light (blue) fronts for $\gamma_{r}=0.4$ and $R e=4000$ (DNS). Continuous lines: DG simulation. Dashed lines: [10] simulation.

and $p=3$ and 2 for $p=4$. Both the Smagorinsky model and the Germano dynamic model have been considered, together with an under-resolved DNS obtained without employing any model. For this case, the simulation could be completed without having to add any artificial diffusion. Notice that approximately 13500 CPU hours were necessary to carry out the DNS at $R e=4000$ until $t=15$. The CPU hours necessary for the LES simulations until $t=20$ with the different turbulence models are shown instead in Table 2.

Table 2. CPU hours necessary for the LES simulations until $t=20$.

\begin{tabular}{cccc}
\hline- & No model & Smagorinsky & Dynamic \\
$p=2$ & 4300 & $3800($ till $t=15)$ & 7200 \\
$p=3$ & 11500 & 12800 & 15300 \\
$p=4$ & 18000 & 26500 & 28000 \\
\hline
\end{tabular}

In Figure 5 we show the dissipated energy as a function of time, obtained with $p=4$, for the different models. The Smagorinsky model (red line) presents a much more 


\section{Bassi, Abbà, Bonaventura, Valdettaro}

dissipative behaviour with respect to the no-model (blue line) and the dynamic model (green line) simulations. This behaviour of the Smagorinsky model is confirmed by the

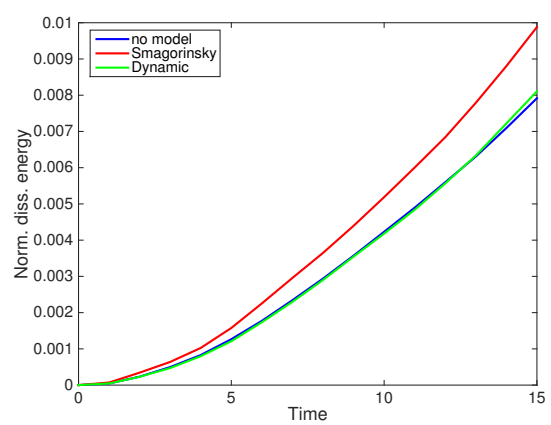

Figure 5. Time evolution of the normalized energies dissipated using no model (blue) the Smagorinsky model (red), the dynamic model (green) for $\gamma_{r}=0.7, R e=40000$ and $p=4$ (LES).

vorticity field at a fixed instant of time $t=15$ (see Figure 8 ). The vorticity field provided by the Smagorinsky model (Figure $8(\mathrm{~b})$ ) is smoother and characterized by less vortical structures. A similar behaviour is also apparent in the results of simulations with $p=2$ and $p=3$ (not shown). Moreover, the vorticity peak values of the Smagorinsky model are lower than the ones obtained with no model and the dynamic model and appear to be less sensitive to the polynomial degree, see table 3. This excessive diffusivity of the Smagorinsky model is in contrast with its almost universal use in the framework of VMS approaches and supports the findings of [7] with respect to the usefulness of more complex subgrid models also in a VMS framework.

Table 3. Vorticity peak values for different models and polynomial degrees at $t=15$.

\begin{tabular}{cccc}
\hline- & No-model & Smagorinsky & Dynamic \\
$p=2$ & {$[-54,57]$} & {$[-24,34]$} & {$[-42,52]$} \\
$p=3$ & {$[-49,68]$} & {$[-38,48]$} & {$[-44,66]$} \\
$p=4$ & {$[-63,77]$} & {$[-42,54]$} & {$[-74,85]$} \\
\hline
\end{tabular}

The precise role played by the dynamic model, however, is not entirely clear in this test case. On one hand, the dynamic model is definitely active and has a growing influence as the polynomial degree (and hence the resolution) decreases. Indeed, the extreme values of $C_{d}$ are bigger for smaller polynomial degrees (see table 4). Moreover, as it can be seen from Figure 6, that shows the elements in which the dynamic constant is smaller than -0.03 , the number of these elements increases as the polynomial degree decreases. On the other hand, as shown in Figure 7, which represents the normalized dissipated energy for the no-model and the dynamic model cases as a function of time in correspondence of different polynomial degrees, the overall impact of the dynamic model appears to be relatively small. The results obtained with different polynomial degrees also clearly show the impact of numerical diffusion at the lower resolutions. It is therefore possible that both the numerical diffusion and the physical dissipation introduced by the dynamical model suppress the energy backscatter induced by the locally negative $C_{d}$, so that the 


\section{LES of gravity currents with high order DG method}

model impact is small in this bulk statistics. The $p=3$ case is the one in which the model appears to have the largest influence, making the dissipated energy quite close to the values obtained for $p=4$. The simulation run with $p=3$ has probably the spatial resolution which is most suitable for LES among those considered here. In the $p=2$ case the numerical dissipation is instead far too large, while in the $p=4$ case the impact of the model appears to be smaller. In this respect, it is also to be remarked that, for $p=4$, the dynamic model yields a total dissipated energy that is larger than that in the corresponding no-model run, as opposed to what happens for lower polynomial degrees, for which the dynamic model always yields lower dissipated energy values. As a conclusion, while some impact of the dynamic model is evident at intermediate resolution, it is clear that the computation of more detailed statistics is necessary to assess more precisely its performance.

Table 4. Minimum and maximum values of the dynamic constant $C_{d}$ at $t=15$.

\begin{tabular}{ccc}
\hline- & $C_{d}^{\min }$ & $C_{d}^{\max }$ \\
$p=2$ & -0.16 & 1.37 \\
$p=3$ & -0.15 & 0.22 \\
$p=4$ & -0.1 & 0.11 \\
\hline
\end{tabular}

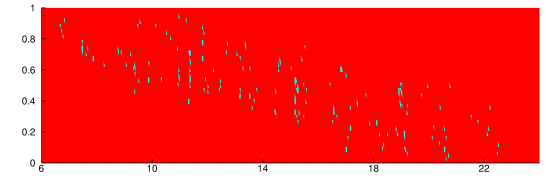

(a)

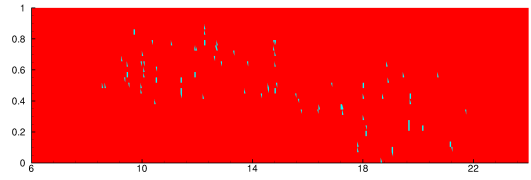

(b)

Figure 6. Elements in which the dynamic constant is smaller than -0.03 . (a) $p 2-p 1$ simulation (b) $p 4-p 2$ simulation. The results are s obtained for $\gamma_{r}=0.7$ and $R e=40000$ at $t=15$.

\subsection{Impact of the numerical flux choice}

In this section, some results obtained with the Rusanov numerical flux, a modified version of the Rusanov flux (where the upwinding velocity is set equal to the velocity of the fluid) and the exact Godunov Riemann solver (implemented as in [20]) will be presented. In particular, in Figure 9 we have the density profiles of a Boussinesq simulation for $R e=44721$ and $\gamma_{r}=0.96$, at $t=5$. We notice that the profile obtained with the Rusanov flux (Figure 9(a)) is highly inaccurate both for the incorrect reproduction of the shape and number of turbulent structures and for the presence of spurious oscillations. These spurious features are instead absent in the profiles obtained with the exact Godunov Riemann solver and with the modified version of the Rusanov flux (Figures 9(b) and 9(c)). This behaviour is probably due to the fact that, when dealing with low Mach number flows, the upwinding velocity in the Rusanov flux is equal to the velocity of sound, which 


\section{Bassi, Abbà, Bonaventura, Valdettaro}

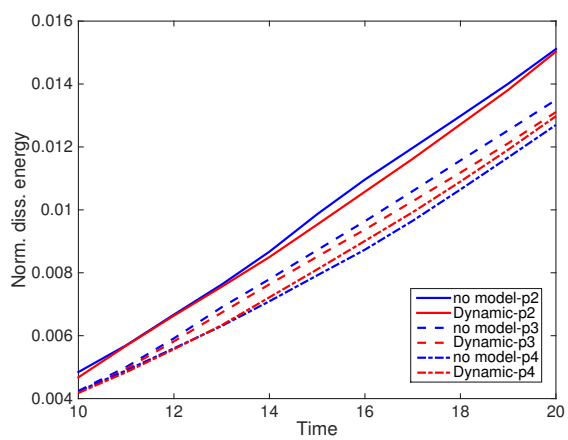

Figure 7. Time evolution of the normalized energies dissipated using no model (blue) and the dynamic model (red) for $\gamma_{r}=0.7$ and $R e=40000$ in the time interval $[10,20]$. Continuous line: $p=2$. Dash line: $p=3$, Dash-dot line: $p=4$.

is very different from the velocity of the fluid in this regime. As a consequence, upwinding is performed with a wrong velocity. This difficulty is indeed overcame by employing the exact solver or simply by substituting the upwinding velocity with the velocity of the fluid. This explanation appears to be consistent with the accurate results obtained by upwind-based, semi-Lagrangian schemes in the simulation of variable density flows with small density differences, see e.g. [26], [27].

\section{Conclusions and future perspectives}

In this paper, we have assessed the capability of a DG-LES model for the numerical simulation of turbulent gravity currents in the non-Boussinesq regime. The results obtained by application of the DG-LES method proposed in [7] have been compared with those presented in [10] in the incompressible case. The quality of our results with respect to [10] has been first assessed using density contours at a fixed instant of time and for different density ratios $\gamma_{r}$. The density contours obtained with our DG simulations appears to be qualitatively similar to the ones in [10] even though some discrepancies are present, especially for the lower density ratio $\gamma_{r}=0.2$.

From a more quantitative viewpoint, two different energy budgets have been considered. First of all, normalized values of potential, kinetic and dissipated energies as a function of time have been compared with the trends reported in [10], finding very good agreement. The temporal evolution of the energy dissipated separately by the light and dense fronts has also been computed. Also in this case, we have quite good agreement with the results in [10], with a good prediction of the more dissipative nature of the dense front with respect to the light one. There is however a slight underestimation of the energy dissipated by the dense front. We can conclude that our approach is able to reproduce quite well the incompressible results of [10] in the low Mach number regime.

Some preliminary LES results have also been presented. Even if these results have not been compared to corresponding DNS results, we can infer that the classic Smagorinsky model is probably too dissipative for the accurate simulation of turbulent gravity currents. This further supports the advantage, first highlighted in [7], of employing dynamical models also in the framework of VMS approaches. On the other hand, even though some evidence was found that dynamical models are active at the resolutions employed, the bulk dissipation rates computed so far are insufficient for a complete assessment of the 


\section{LES of gravity currents with high order DG method}

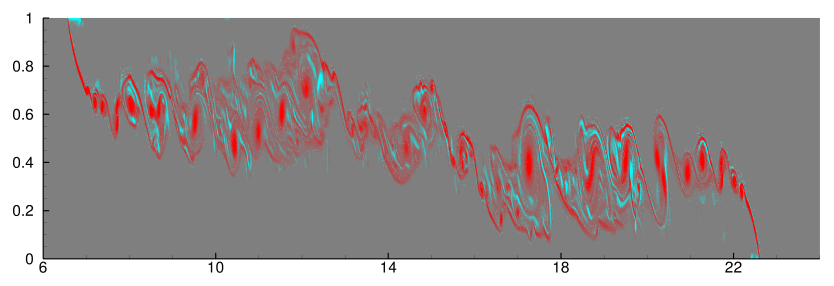

(a)

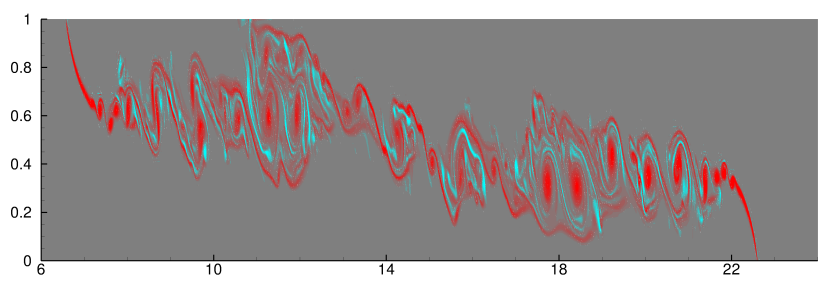

(b)

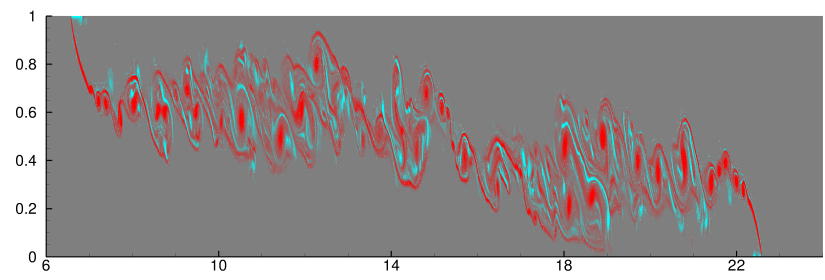

(c)

Figure 8. Vorticity field obtained for $\gamma_{r}=0.7, R e=40000$ and $p=4$ (LES) at $t=15$ Red: positive values. Blue: negative values. (a) No model, value range $[-63,77]$. (b) Smagorinsky model, value range $[-42,54]$. (c) Dynamic model, value range $[-74,85]$.

performance of different models. It will be therefore necessary to compute additional diagnostics, like the available potential energy, see [28], [29], [5], [4], [30], or the local structure function criterion employed in [31] for polynomial degree adaptation. Finally, concerning the impact of the numerical flux choice, we can conclude that the generally used Rusanov flux may not be the best option for variable density, very low Mach number regimes.

From the physical point of view, the next planned step is to carry out Large Eddy Simulations employing also more advanced models, like the anisotropic dynamic model proposed in [32] and modeling the subgrid stresses by the novel proposals in [33] for compressible, variable density flows. From the computational point of view, we are aware that employing an explicit time integration method in presence of low Mach numbers leads to computational inefficiency; we are indeed working on the implementation of a semiimplicit time integration method in order to perform also 3D simulations. Furthermore, a deeper investigation of the influence of the numerical flux on the solutions in variable 


\section{Bassi, Abbà, Bonaventura, Valdettaro}

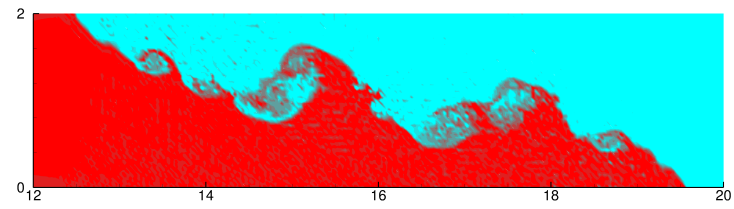

(a)

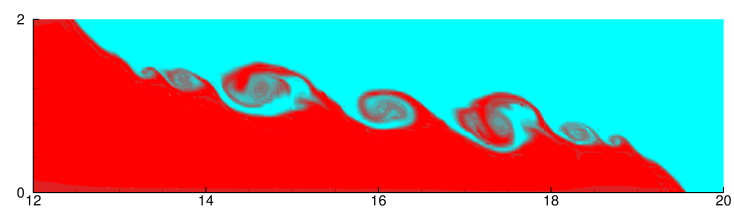

(b)

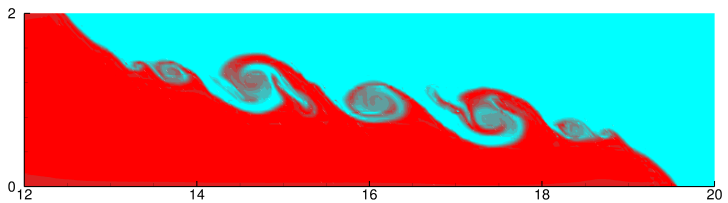

(c)

Figure 9. Density for $R e=44721$ and $\gamma_{r}=0.96$ at time $t=5$. (a) Rusanov flux. (b) Godunov exact Riemann solver. (c) Modified Rusanov flux (upwinding velocity equal to the velocity of the fluid)

density low-Mach number would be required to better understand some of the results presented in this paper.

\section{Acknowledgements}

This paper contains an extended version of results presented by the first author at the 2016 SIMAI Congress and is part of the first author's PhD thesis work. We are happy to acknowledge the continuous help of M. Restelli and M.Tugnoli with the application of the FEMILARO code. Several useful discussions with T. Esposti Ongaro and M. Cerminara are also kindly acknowledged, along with the useful comments of the reviewer, which greatly helped in improving the original version of the paper. The results of this research have been achieved using the computational resources made available at CINECA (Italy) by the LISA high performance computing project DECLES: Large Eddy Simulation of Density Currents and Variable Density Flows, HPL13PJ6YS. 


\section{LES of gravity currents with high order DG method}

\section{REFERENCES}

1. W. Houf and R. Schefer. Analytical and experimental investigation of small scale unintended releases of hydrogen. International Journal of Hydrogen Energy, 33:14351444,2008 .

2. A.E. Gill. Atmosphere-Ocean Dynamics. Academic Press, 1982.

3. J.F. Louis. A parametric model of vertical eddy fluxes in the atmosphere. Boundary Layer Meteorology, 17:197-202, 1979.

4. L.C. Berselli, P.F. Fischer, T. Iliescu, and T.M. Özgökmen. Horizontal Large Eddy Simulation of Stratified Mixing in a Lock-Exchange System. Journal of Scientific Computing, 49:3-20, 2011.

5. T.M. Özgökmen, T. Iliescu, and P.F. Fischer. Large Eddy Simulation of stratified mixing in a three-dimensional Lock-exchange system. Ocean Modelling, 26:134-155, 2009.

6. T.M. Özgökmen, T. Iliescu, P.F. Fischer, A. Srinivasan, and J. Duan. Large Eddy Simulation of stratified mixing in two-dimensional dam-break problem in a rectangular enclosed domain. Ocean Modelling, 16:106-140, 2007.

7. A. Abbà, L. Bonaventura, M. Nini, and M. Restelli. Dynamic models for Large Eddy Simulation of compressible flows with a high order DG method. Computers \& Fluids, 122:209-222, 2015.

8. T.J.R. Hughes, A.A. Oberai, and L. Mazzei. Large eddy simulation of turbulent channel flows by the variational multiscale method. Physics of Fluids, 13:1784-1799, 2001.

9. V. John and A. Kindl. Numerical studies of finite element Variational Multiscale Methods for turbulent flow simulations. Computer Methods in Applied Mechanics and Engineering, 199:841-852, 2010.

10. V. K. Birman, J.E. Martin, and E. Meiburg. The non-Boussinesq Lock-exchange problem. Part 2. High-resolution simulations. Journal of Fluid Mechanics, 537:125$144,2005$.

11. H. Schlichting. Boundary-layer theory. 7 th edition. McGraw-Hill, 1979.

12. P. Sagaut. Large Eddy Simulation for Incompressible Flows: An Introduction. Springer Verlag, 2006.

13. E. Garnier, N. Adams, and P. Sagaut. Large Eddy Simulation for Compressible Flows. Springer Verlag, 2009.

14. M. Germano, U. Piomelli, P. Moin, and W.H. Cabot. A Dynamic Subgrid-Scale Eddy Viscosity Model. Physics of Fluids, 3(7):1760-1765, 1991.

15. F.X. Giraldo and M. Restelli. A study of spectral element and discontinuous Galerkin methods for the Navier-Stokes equations in nonhydrostatic mesoscale atmospheric modelling: equation sets and test cases. Journal of Computational Physics, 227:3849$3877,2008$.

16. D. N. Arnold, F. Brezzi, B. Cockburn, and L.D. Marini. Unified analysis of Discontinuous Galerkin methods for elliptic problems. SIAM Journal of Numerical Analysis, 39:1749-1779, 2002. 


\section{Bassi, Abbà, Bonaventura, Valdettaro}

17. F. Bassi and S. Rebay. High Order Accurate Discontinuous Finite Element Method for the Numerical Solution of the Compressible Navier-Stokes Equations. Journal of Computational Physics, 131:267-279, 1997.

18. P. Castillo, B. Cockburn, I. Perugia, and D. Schötzau. An a priori analysis of the Local Discontinuous Galerkin method for elliptic problems. SIAM Journal of Numerical Analysis, 38:1676-1706, 2000.

19. B. Cockburn and C.W. Shu. The Local Discontinuous Galerkin Method for TimeDependent Convection Diffusion Systems. SIAM Journal of Numerical Analysis, 35:2440-2463, 1998.

20. J.J. Gottlieb and C.P.T. Groth. Assessment of Riemann Solvers for Unsteady OneDimensional Inviscid Flows of Perfect Gases. Journal of Computational Physics, 78:437-458, 1988

21. R. Cools. An Encyclopaedia of Cubature Formulas. Journal of Complexity, 19:445453, 2003.

22. S. S. Collis. Discontinuous Galerkin methods for turbulence simulation. In Proceedings of the 2002 Center for Turbulence Research Summer Program, pages 155-167, 2002.

23. S. S. Collis and Y. Chang. The DG/VMS method for unified turbulence simulation. AIAA paper, 3124:24-27, 2002.

24. F.van der Bos, J.J.W. van der Vegt, and B.J. Geurts. A multi-scale formulation for compressible turbulent flows suitable for general variational discretization techniques. Computer Methods in Applied Mechanics and Engineering, 196:2863-2875, 2007.

25. FEMilaro, finite a toolbox. https://bitbucket.org/mrestelli/femilaro/wiki/Home. Available under GNU GPL v3.

26. L. Bonaventura. A semi-implicit, semi-Lagrangian scheme using the height coordinate for a nonhydrostatic and fully elastic model of atmospheric flows. Journal of Computational Physics, 158:186-213, 2000.

27. G. Tumolo and L. Bonaventura. A semi-implicit, semi-Lagrangian, DG framework for adaptive numerical weather prediction. Quarterly Journal of the Royal Meteorological Society, 141:2582-2601, 2015.

28. K.B. Winters, P.N. Lombard, J.J. Riley, and E.A. D'Asaro. Available potential energy and mixing in density-stratified fluids. Journal of Fluid Mechanics, 289:115-128, 1995.

29. Y. Tseng and J.H. Ferziger. Mixing and available potential energy in stratified flows. Physics of Fluids, 13:1281-1293, 2001.

30. L.C. Berselli, M. Cerminara, and T. Iliescu. Disperse Two-Phase Flows, with Applications to Geophysical Problems. Pure and Applied Geophysics, 172:181-196, 2015.

31. M. Tugnoli, A. Abbà, L. Bonaventura, and M. Restelli. A locally $p$-adaptive approach for Large Eddy Simulation of compressible flows in a DG framework. MOX Report 37/2016, MOX - Politecnico di Milano, 2016.

32. A. Abbà, C. Cercignani, and L. Valdettaro. Analysis of Subgrid Scale Models. Computer and Mathematics with Applications, 46:521-535, 2003.

33. M. Germano, A. Abbà, R. Arina, and L. Bonaventura. On the extension of the eddy viscosity model to compressible flows. Physics of Fluids, 2014. 